\title{
Event related potentials and serial list picture memory in Parkinson's patients
}

\author{
M.S. Korsnes and I. Reinvang \\ Neuropsychological Laboratory, Department of Psychosomatic and Behavioural Medicine, \\ The National Hospital, Oslo, Norway
}

Correspondence to: M.S. Korsnes, Neuropsychological Laboratory, Department of Psychosomatic and Behavioural Medicine, The National Hospital, 0027 Oslo, Norway

\begin{abstract}
Two experiments examined short-term memory for order information in six patients diagnosed with Parkinson's disease (PD) and six control subjects while event related potentials (ERPs) were recorded. The subjects were tested for recognition of abstract spatial designs and words after a $5 \mathrm{~s}$ retention interval. The PD patients failed to respond in $29 \%$ of all trials, but the overall accuracy was similar to that in the control group when these trials were excluded. The corresponding ERP results show serial position variations both after presentation of the probe items, and after presentation of the memory set items. The amplitudes were generally lower at all positions for the PD patients at the parietal midline electrode, and the amplitudes were similar for both groups at the frontal electrode. Also, the ERP latencies were significantly slower for the PD patients than for the control group at all conditions. Indirectly the data are consistent with an interpretation of cognitive deficit in PD stressing attention resources.
\end{abstract}

Keywords: Event related potentials - Serial list - Recognition memory - Parkinson's disease

\section{INTRODUCTION}

Human memory for a list of items is better for the first and last items than it is for the middle items, giving rise to the $\mathrm{U}$-shaped list position function. The phenomenon that the first and last items in a series of learned acts will be remembered better than middle items, is referred to as 'primacy' and 'recency' effects respectively (Kausler, 1991). List position designs represent well established methods for assessment of performance differences in short-term memory. Studies have concluded (Craik, 1968; Korsnes and Gilinsky, 1993; Korsnes and Magnussen, 1996; Lewandosky and Murdock, 1989; Wright et al., 1985) that basic memory processes producing the list position functions are similar for young and older healthy subjects, and even for monkeys and pigeons.

Previous reports have concluded that Parkinson's disease (PD) affects memory (e.g. Reitan and Boll, 1971), but several studies using standardized scales such as the Wechsler Memory Scale, or standard tests (for example digit span, recall of stories) have shown either a marginal deficit, or no deficit in parkinsonian subjects (Cooper et al., 1992; Talland, 1969). If there is a memory deficit it must therefore involve selective aspects of memory. Studies have reported findings indicating deficits in short-term

(c) 1996 Rapid Science Publishers working memory, in recall (as opposed to simple recognition), or in memory for certain types of information (visual/visuo-spatial information, or information on time relations). Studies reporting memory impairments for verbal and non-verbal material have found them to be especially prominent for shortterm memory compared with long-term memory (Sahakian et al., 1988).

Sagar et al. (1988) found impaired recency discrimination of sequentially presented words in PD patients but preserved content recognition. The patients were asked to identify which one of two nouns had been presented before in a long list, or the most recent of two nouns. The impaired recency discrimination was particularly pronounced at short retention intervals. The results were later confirmed by Cooper et al. (1991), with the addition that the deficits were more prominent in chronic medicated patients. Della Sala et al. (1987), using free recall tests and verbal and spatial span tests, did not find an impaired recency effect in PD that would be expected on the basis of impaired recency discrimination (Sagar et al., 1985, 1988; Cooper et al., 1991). On the contrary they found that primary memory was impaired in PD. Cooper et al. (1993) did not find evidence of a generalized temporal ordering deficit for verbal 
information in PD patients. The contradicting conclusions on the recency effect by Sagar et al. (1988) and Della Sala et al. (1987) indicate that memory for temporal ordering in PD is of particular interest, and should be explored further with serial list tests. PD patients may have more general deficits in memory and attention, which could complicate interpretation of further studies, and the format for testing memory (recall or recognition; visual versus verbal material) may influence the results. A recent study (Breen, 1993) investigated verbal short-term serial list memory functions in PD patients and found impairment in recall for verbal material, but not in recognition. Flowers et al. (1984) suggested that the reason why impairments are found in recall tasks and not in recognition tasks is the passive nature of the recognition tasks where only a simple decision is necessary which may be performed correctly even on the basis of degraded information.

Visual cognitive abnormalities are frequently reported in PD (Boller et al., 1984), but their occurrence is not correlated with motor impairment (Cooper et al., 1992). Previous studies have indicated deficits in several aspects of visual processing. Bradley et al. (1989) suggest that speed of information processing deficits may be especially prominent on visuo-spatial tasks, and they concluded that the visuo-spatial subsystem of working memory is impaired in PD patients. Also, Taylor et al. (1986) found deficits in recognition of spatial position of items among nondemented PD patients when tested after a delay. Further evidence for specific visual memory deficits was found by Sullivan and Sagar (1989), who described deficits in memory for abstract designs among both demented and non-demented PD patients. Overall the demented patients were more severely impaired, but both patient groups demonstrated relatively poorer memory at short rather than longer retention intervals compared with the control group. Levin et al. (1989) found PD patients to perform comparably to normal subjects on attentional and visuo-spatial measures, but they were impaired in recognition of faces, and other figurative memory measures. They also reported impairment in verbal memory. However, visual cognitive abnormalities are often related to other cognitive impairments, so it is not clear if these results provide evidence of specific deficits in visuo-spatial memory. Della Sala et al. (1986) did not find evidence for a generalized visuo-spatial deficit in $\mathrm{PD}$, and suggested that test biases or sample biases could account for positive findings.

ERP (event related potentials) methodology is a valuable addition to behavioural methods in the study of memory, because it reflects the processing of stimuli not associated with behavioural response. This is of particular importance in the study of a group that suffers from movement deficits, that might influence responses based on skill or on the basis of time to completion. Studies of verbal recall and recognition in healthy subjects have identified ERP markers of stimulus repetition (Rugg et al., 1994), and of study items that are more likely to be successfully recalled or recognized (Paller et al., 1988). Paller et al. $(1987,1988)$ found "ERP differences based on subsequent memory performance" $(\mathrm{Dm})$ in recognition and recall of words, by comparing the ERPs resulting from a distractor task (rating words as "interesting or uninteresting", or "edible or inedible") with a free recall task, and later a yes/no recognition test, including the words from the distractor test. Begleiter et al. (1993) studied visual short-term memory for simple and complex abstract designs and found evidence of memory-related ERP markers in a stimulus matching task. They found "visual memory potentials" (VMPs) between sample trials and matching trials, and between matching and non-matching conditions. The VMPs were particularly strong between sample and matching simple stimuli. Patterson et al. (1991) used a standard recognition task (five item serial list) on healthy subjects while recording ERPs simultaneously. They found electrophysiological evidence of list position effects in short-term memory, and significant recency effects in the average amplitudes of a late positive parietal potential for auditory digits, but not for visual stimuli. Amplitudes for both the mid-list items and the last list items decreased relative to the amplitude for the first list item for both visual and auditory stimuli. Several studies have found various effects of ERPs in PD patients (reviewed in Amabile et al., 1990) including abnormal visual evoked potential, latency abnormalities of auditory evoked potentials, generally lower amplitudes in PD and a delayed P3 latency compared to normal subjects. Amabile et al. (1990) suggest a dopaminergic effect on the neural generator of P3, and that the delayed P3 might relate to a deficit in the dopaminergic system, because they observed a generally faster P3 in healthy subjects and PD patients after intake of L-dopa medication.

The present study aimed to investigate serial position functions in short-term visual memory for PD patients. We applied a recognition task that requires multiple choice which is more demanding than traditional recognition tests, and memory performance and visual evoked potentials of the list position functions were recorded, examining the effect of two types of visual stimuli (abstract spatial patterns versus

70 Behavioural Neurology . Vol 9 . 1996 
words). The hypothesis was that abnormal primacy/ recency functions would be found based on previous reports of deficits in temporal order discrimination (Sagar et al., 1988) and primary memory (Della Sala et al., 1987). ERP recordings can provide information regarding processing differences, and thus help to resolve the cognitive locus of any list position deficits. Comparing ERPs at time of presentation of memory set items and at the recognition probe may reveal physiological differences in encoding or memory representation, and relate to the controversy on the contribution of encoding versus retrieval deficits to the observed memory performance deficit in PD patients.

\section{METHODS}

\section{Subjects}

Six PD patients were recruited from the National Hospital Oslo, five men and one woman with a mean age of 46.3 years (33-55) and a mean education of 13 years. The PD patients were refunded for travelling expenses. The control group were five men and one woman, with a mean age of 39.6 years $(30-45)$ and a mean education of 16 years. The age differences within the two groups were not considered to cause ERP differences. In a study by Karayanidis et al. (1993), the average ERP latency advantage for a group with an average age of 27 years was only $10 \mathrm{~ms}$ over a group of an average age of 46 years. Because the same subjects participated in both experiments, the order in which each subject received the tasks was randomized between experiments, and counterbalanced between subjects. All patients were clinically diagnosed with Parkinson's disease, and no patient had undergone thalamotomy or other surgical procedures for relief of parkinsonian symptoms. Four were newly diagnosed and unmedicated, and two had 10 and 7 years' disease duration and were on dopamine medication. The PD patients were not clinically demented and did not show general intellectual impairment on a neuropsychological battery.

\section{ERP-recordings}

The recordings were made with 19 channels of EEG and two channels for recording eye movement artifacts, with bipolar recordings from the canthi of each eye, and from the supraorbital ridge and suborbital region of the left eye. Signals were referred to linked ears, and impedances were kept below $10 \mathrm{kOhm}$. The EEG leads were placed in accordance with the international 10-20 system and in addition to frontal $(\mathrm{FZ})$, central $(\mathrm{CZ})$, and parietal midline (PZ), in- cluded FP1, FP2, F3, F4, F7, F8, T3, T4, C3, C4, T5, T6, P3, P4, O1, and O2. Only data from FZ, CZ and $\mathrm{PZ}$ are included in the present report. Single sweeps from 200 pre- to $1200 \mathrm{~ms}$ post-stimulus were recorded and with filter settings 0.16 to $35 \mathrm{~Hz}(24 \mathrm{~dB}$ attenuation). Statistical eye-movement correction with the procedure described by Semlitsch et al. (1986) was applied to single sweeps before averaging, and single sweeps with amplitude $>100$ or $<-100 \mu \mathrm{V}$ on EEG channels were rejected. Stimulus presentation, data recording and analyses were performed on two $48633 \mathrm{MHz}$ PCs with Neuroscan software.

\section{Stimuli}

The stimuli in condition 1 consisted of 384 words, in 64 presentation sequences and probe pictures depicting meanings of the words. Each trial contained four trial-unique inspection items and a picture that symbolized one of them (recognition control probe item) to be tested for recognition (example in Fig. 1a). The probes were designed so that the images were prototypical mental images, easily associable with the corresponding word. The methodological design developed in Korsnes et al. (1996) avoids using distractor tasks which may suppress the recency effect (Glanzer and Cunitz, 1966). The subjects were instructed not to rehearse the words vocally or sub-vocally.

The stimuli in condition 2 consisted of 384 abstract spatial designs used in presentation sequences consisting of 16 series of four items (example in Fig. 1b). The presentation format was identical to that of condition 1 , but the probe recognition item was identical to one of the memory set items. The abstract spatial patterns were retrieved with more difficulty than the words at all ordinal positions in normally functioning young and older adults (Korsnes and Magnussen, 1996).

\section{Procedure}

The computer screen was placed $75 \mathrm{~cm}$ from the subject. The series were manipulated by the experimenter from an adjacent control room and presented on the screen in front of the subject. The stimuli were projected at the centre of the screen, subtending a visual angle of $7.6^{\circ}$. Series of four visual images were presented, one visual image at a time with an interstimulus interval of $1 \mathrm{~s}$ and $500 \mathrm{~ms}$ exposure time. The unfilled delay intervals between exposure to the fourth and last inspection item and the probe was $5 \mathrm{~s}$. An auditory signal warned the subject $2 \mathrm{~s}$ before the probe was displayed. Subjects responded to each recognition probe by pressing the appropriate button on a four button indicator panel, indicating list position (1-4). All recognition probes were positive 


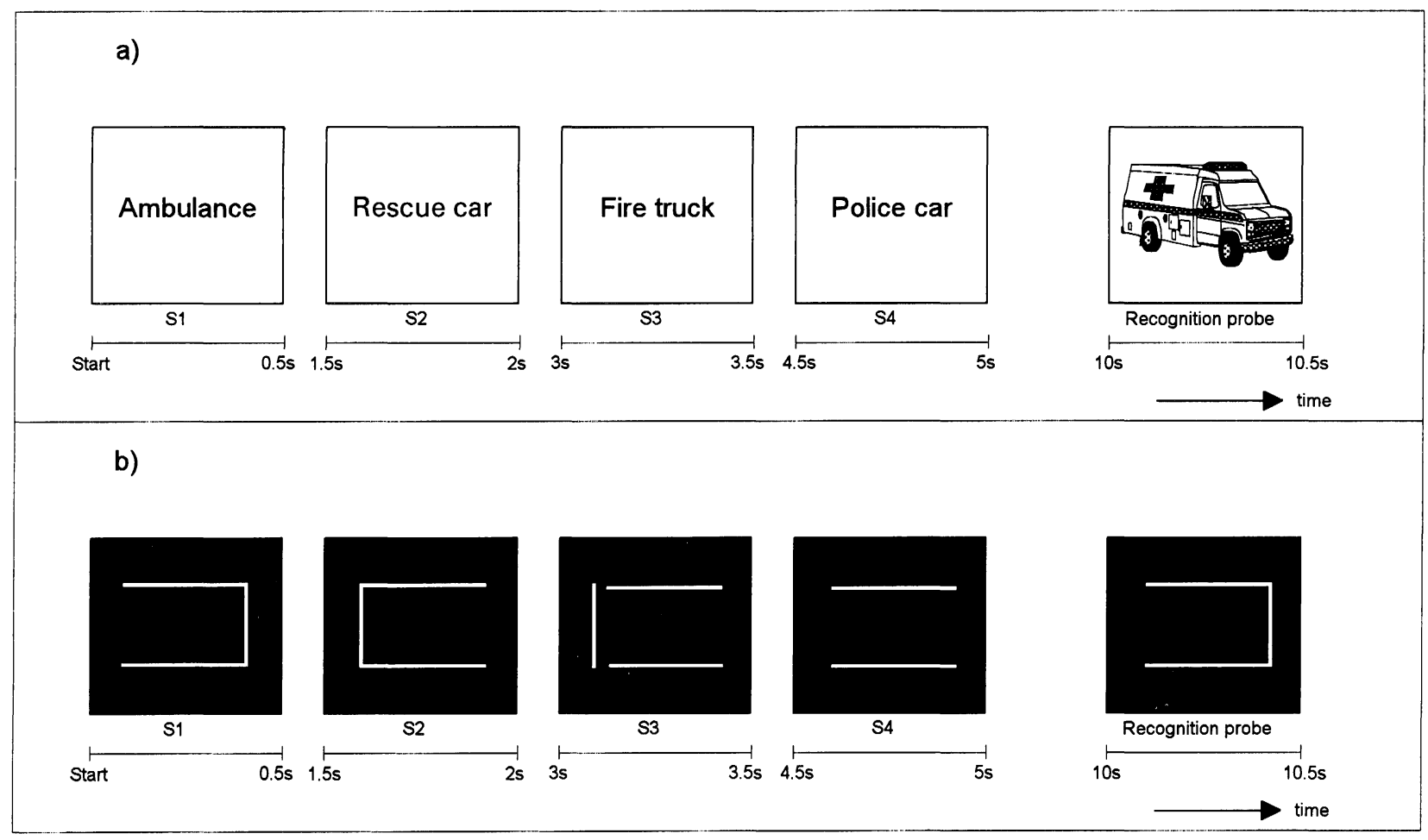

FIG. 1. Sample series for the words (the words were presented in Norwegian) and for the spatial abstract designs.

probes. The subjects had 64 trials and the stimuli were randomized for each subject, at each list position. If the subject failed to respond within $5 \mathrm{~s}$, the response was not registered.

\section{Analyses of ERP components}

For each stimulus type, ERPs for correct responses, and the percentage correct responses were averaged according to their position (1-4) in the memory set, yielding four separate averages for each stimulus type of each measure. Because 64 trials were presented, 16 trials represented each list position for each subject. Measures of baseline to peak amplitudes were performed on the averaged wave form for both the probes and the memory set items. Amplitudes were measured relative to the mean voltage during the pre-stimulus baseline period. Analyses of ERP components were guided by previous research on similar paradigms (Patterson et al., 1991; Pratt et al., 1989) and on the wave forms actually observed (Figs 3 and 6). When ERPs are collected in the memory scanning task, the probe is associated with a sustained late positive potential overlapping a P300 complex (Gomer et al., 1976). Although the patients in the present experiment showed sustained positivity in the time window previously described, the onset or offset of distinctive positive components could not be clearly differentiated. The decision to analyse P3 and a later positive component was therefore partly based on a priori considerations (see below).

\section{Data analyses}

Missed trials (subjects did not press any button within the time limit) were subtracted from the total number of trials at each position, and the average number correct was calculated. ERPs were analysed for each stimulus type for both the $\mathrm{FZ}, \mathrm{CZ}$ and $\mathrm{PZ}$ electrode locations using multivariate analyses of variance (MANOVA) procedures with group, stimulus type, electrode and position as factors. Separate MANOVAs were also performed for each group and at each condition. Significance level was set at $p<0.05$.

\section{RESULTS}

\section{Accuracy}

The position/accuracy curves for the two conditions are shown as Fig. 2. The PD patients failed to respond within the available $5 \mathrm{~s}$ in $29 \%$ of all trials evenly distributed at all list positions and between stimuli conditions, and the control group in 3\% of all trials. These no-response trials are not included in Fig. 2.

72 Behavioural Neurology . Vol $9 \cdot 1996$ 


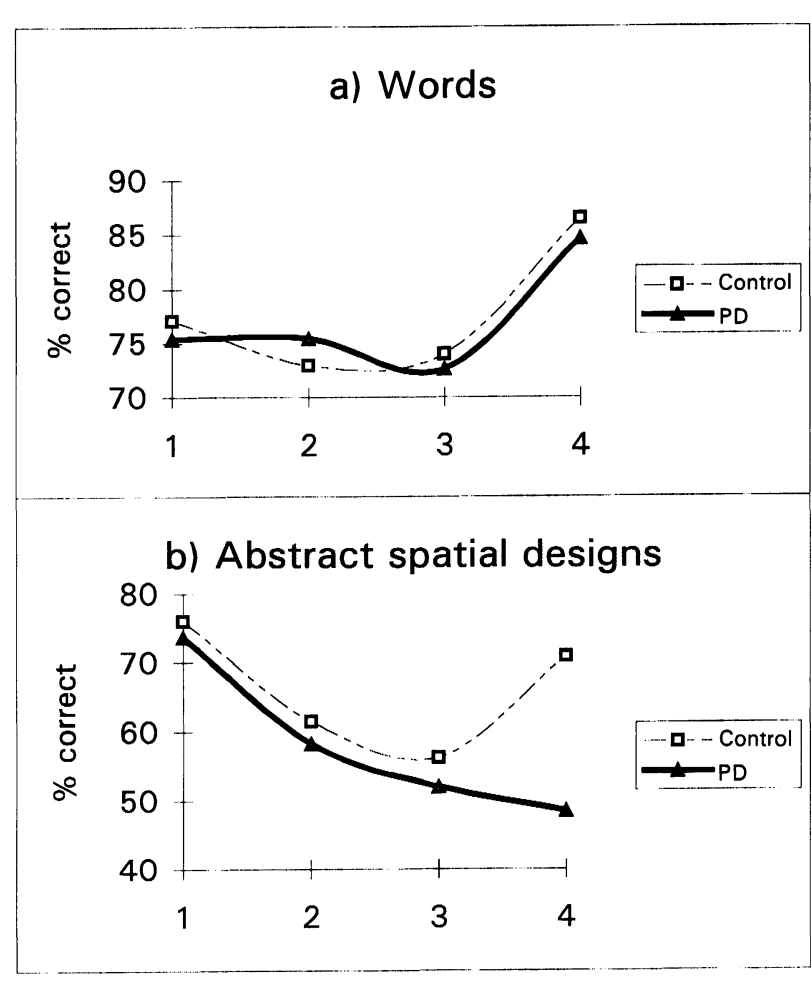

FIG. 2. Accuracy results for the control group and the PD patients for a) words and b) abstract spatial designs.

A MANOVA with repeated measures on list position and averaged tests of significance (Group $\times$ Stimulus $\times$ Position) showed significant main effects of stimulus conditions $[\mathrm{F}(1,10=21.29, p<0.01]$ and of position $[\mathrm{F}(3,30)=4.15, p=0.01]$. There were no significant main effects of subject group $[\mathrm{F}(1,10)=1.7, p>0.2)$, or the interactions involving subject groups [Group $\times$ Position: $F(3,30)=1.02$, $p>0.3$; Stimulus $\times$ Group $\times$ Position: $\mathrm{F}(3,30)=$ $1.07, p>0.3]$. A separate MANOVA on the abstract spatial designs did not show significantly different list position results for the two groups, even though the curves are deviant on the last list position [Group: $\mathrm{F}(1,10)=2.8, p>0.1$; Group $\times$ Position: $\mathrm{F}(3,30)=$ 1.76, $p>0.1$; Pos. 4, Group: $F(1,10)=4.01$, $p>0.05$ ], because of unstable results at Pos. 4 for the PD patients (Mean: 7.5, SD 4.0). It is therefore not conclusive that there was a recency deficit in PD for the spatial abstract designs.

\section{ERP to probe items}

The ERP to probe items are presented in Fig. 3. The grand averages suggest a positive complex with peak latency about 300-400 ms after stimulus onset, and a suggested later positivity interacting with the downward slope of the first positivity. It was decided on an a priori basis to analyse an early component

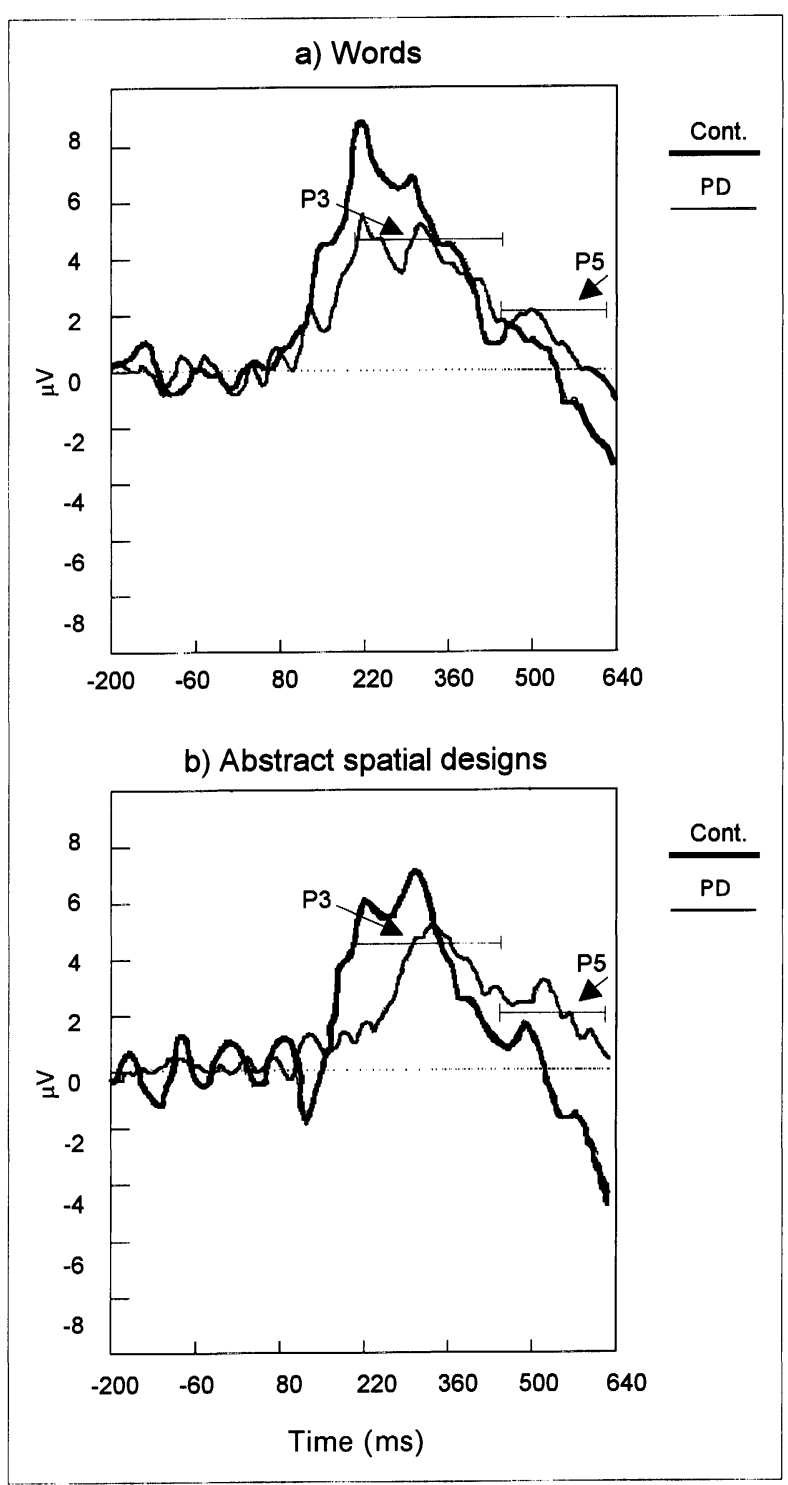

FIG. 3. Average ERP amplitudes for the two groups under the two conditions recorded after presentation of the probe (PZ).

corresponding to conventional criteria for P300 (P3), and a later component following the P300 and lasting for the duration of the observed positivity on the grand average. This latter peak (labelled descriptively as P5) was assumed to be more likely to reflect memory-specific processing (Paller et al., 1987, 1988). Paller et al. (1987, 1988) found ERPs for words to differ as a function of later memory performance in the range from 400 to $800 \mathrm{~ms}$ after stimulus onset, for recall and recognition tasks. The time windows used for evaluating the ERPs were decided from the grand average curves in Fig. 3, and the maximum amplitudes within the window were scored. Because 


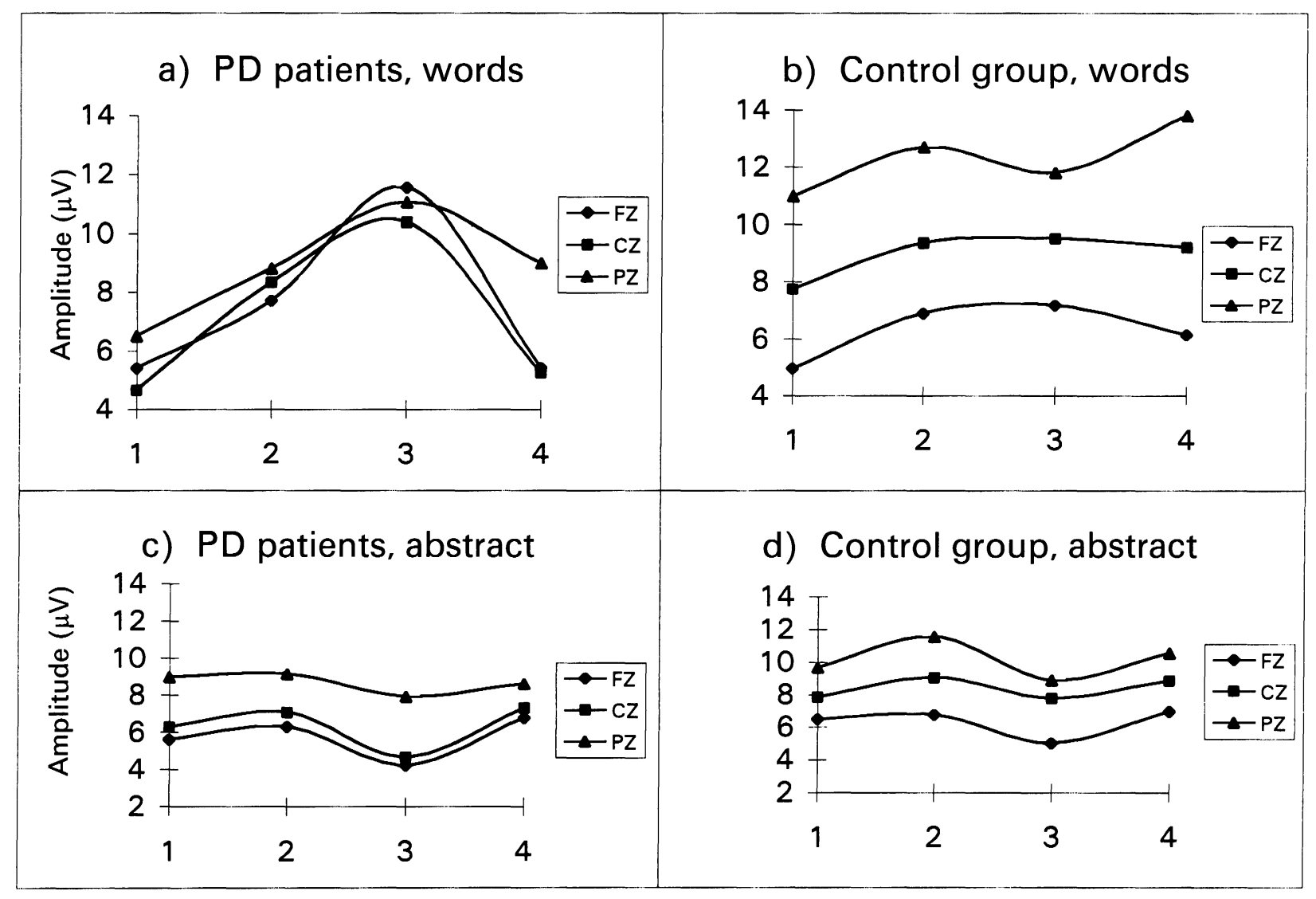

\section{List Position}

List Position

FIG. 4. ERPs to probes at $P 3$ for the two groups under the two conditions. The highest positive amplitude within a window from $200-470 \mathrm{~ms}$ was recorded.

there were ERP latency differences between the groups, the time windows for analysis were defined with wide margins to take account of the variation in both groups [P3 (probes) $=200-470 \mathrm{~ms} ; \quad \mathrm{P5}$ (probes) $=450-630 \mathrm{~ms}$, P3 (memory set items) $=$ $200-450 \mathrm{~ms}]$.

For the P3 (200-470 ms) ERP components of the probe recognition items a MANOVA with repeated measures and averaged tests of significance (Group $\times$ Stimulus $\times$ Position) showed a main serial position effect $[\mathrm{F}(3,30)=3.59, p<0.05]$, but not main effects between the groups [Group: $\mathrm{F}(1,10)=2.79, \quad p>0.09), \quad$ Group $\times$ Position: $\mathrm{F}(3,30)=1.84, p>0.1]$. However, the serial position results were different for the two stimulus conditions [Group $\times$ Stimulus $\times$ Position: $\quad F(3,30)=2.79$, $p<0.05]$. Separate MANOVAs showed similar results at the two conditions for the control group, but differences for the PD patients, with higher amplitudes for the mid-list positions for words and similar amplitudes for all positions for spatial abstract designs $[P D$, Stimulus $\times$ Position: $F(3,30)=7.06$, $p<0.01$; Control, Stimulus $\times$ Position: $\mathrm{F}(3,30)=$ 2.21, $p>0.05$.

A similar MANOVA for the P5 component (450$630 \mathrm{~ms}$ ) showed a significant amplitude difference between the list positions $[\mathrm{F}(3,30)=4.72, p<0.01]$, amplitude differences between the groups $[\mathrm{F}(1,10)=$ 7.78, $p<0.01$ ], and a different amplitude pattern at list positions between the two groups [Group $\times$ Position: $\mathrm{F}(3,30)=4.00, p<0.01]$. The differences in serial position results between the two stimulus conditions were not significant [Group $\times$ Stimulus $\times$ Position: $\mathrm{F}(3,30)=1.24, p>0.3]$.

The list position results between the groups at the two peaks were similar, even though amplitude levels and serial position results at each peak were different, as indicated by a MANOVA [Peak $\times$ Group: $F(1,10)$ $=10.22, p<0.01$; Peak $\times$ Position: $\mathrm{F}(3,30)=2.81$, $p<0.05$; Peak $\times$ Group $\times$ Position: $\mathrm{F}(3,30)=1.48$,

74 Behavioural Neurology . Vol 9 . 1996 


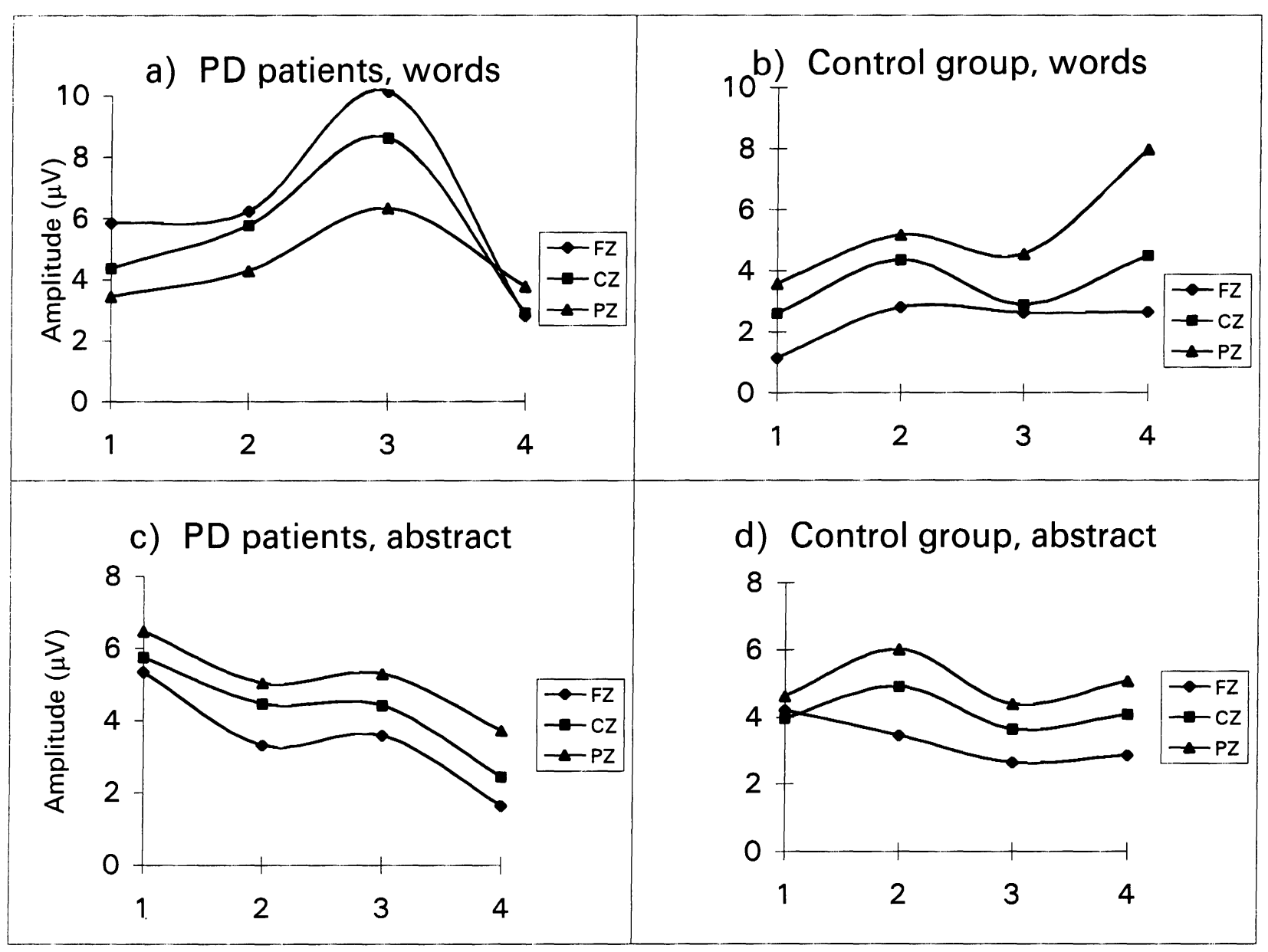

List Position

List Position

FIG. 5. ERPs to probes at P5 for the two groups under the two conditions. The highest positive amplitude within a window from $450-630 \mathrm{~ms}$ was recorded.

$p>0.2)$. The Peak $\times$ Group interaction is due to lower P3 amplitudes in PD, whereas P5 amplitudes in $\mathrm{PD}$ are similar or higher than for the control subjects.

\section{ERP to memory set items}

The finding of a significant position effect on the ERP after presentation of the probes, raised the question of whether the same effects are found at encoding. Grand average ERPs to memory set items are displayed in Fig. 6.

The peak amplitude for P3 (200-450 ms) was measured and analysed with reference to list position, combining the two mid-positions. The results in Fig. 7 show lower amplitudes for the PD patients.

A MANOVA showed a main effect of list position $[\mathrm{F}(2,20)=5.68, p<0.01]$, and a confirmation of the visual inspection of an amplitude difference between the groups $[\mathrm{F}(1,10)=31.42, p<0.01]$, and different serial position characteristics between the groups $[\mathrm{F}(2,20)=4.47, p<0.05]$. There are amplitude differences between the stimulus categories $[F(1,10)=$ 5.13, $p<0.05]$, but these do not interact with list position [Stimuli $\times$ Position: $\mathrm{F}(2,20)=0.18, p>0.8$; Group $\times$ Stimuli $\times$ Position: $\mathrm{F}(2,20)=0.38, \quad p>$ $0.6]$.

\section{Group differences in ERP between FZ, CZ and PZ}

A general similarity for P3 amplitudes was found at all electrodes for the PD patients, but for the control group the amplitudes at FZ were lower than at $\mathrm{CZ}$ and the amplitudes at $\mathrm{PZ}$ were highest. General factorial ANOVAs (Group $\times$ Stimuli $\times$ Electrode) showed this amplitude difference between electrodes after presentation of the memory set items [Group $\times$ 


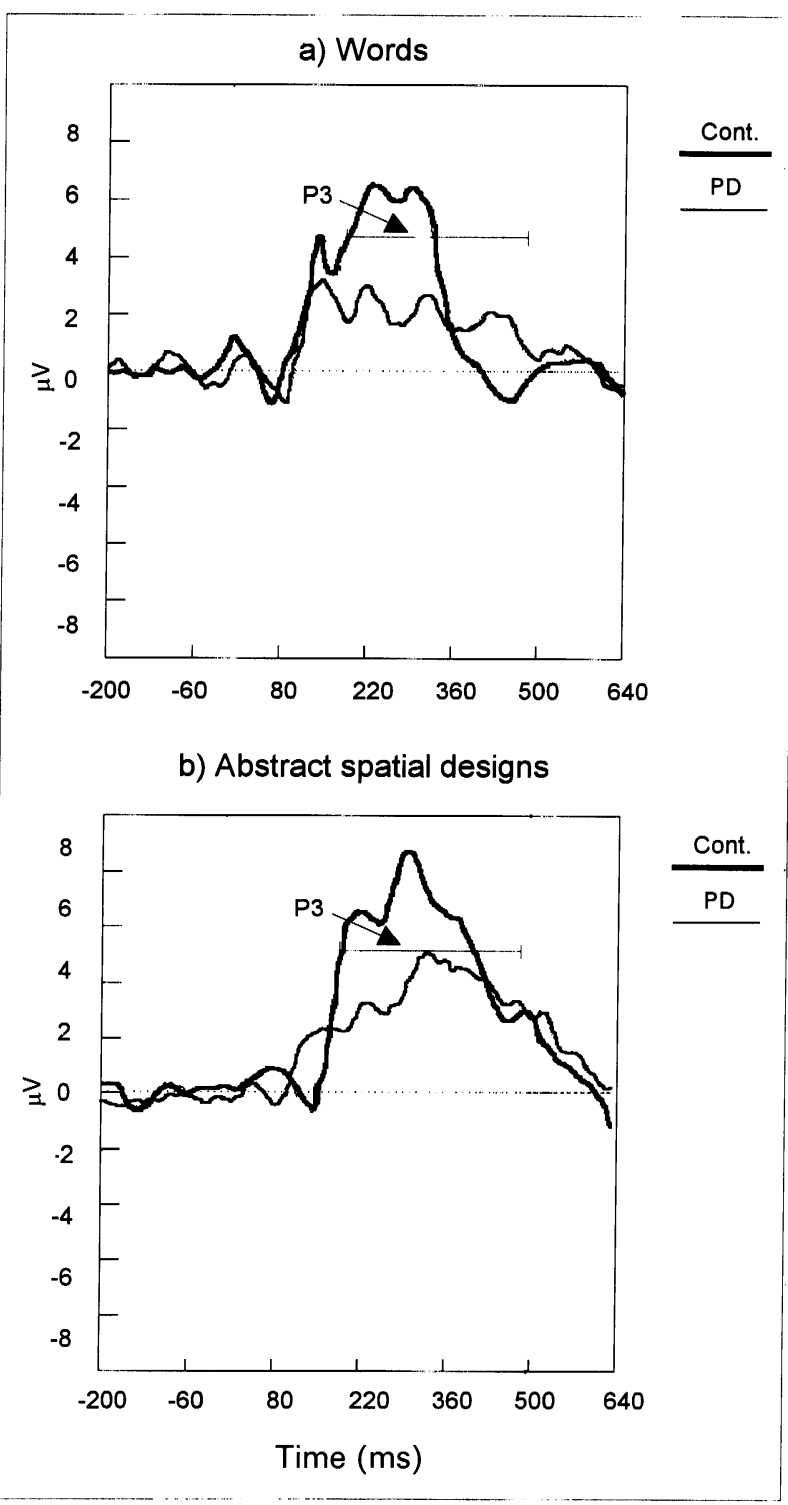

FIG. 6. Average ERP amplitudes at $P Z$ for the two groups under the two conditions recorded after presentation of the memory set items (PZ).

Electrode: $\quad \mathrm{F}(2,20)=5.9, \quad p<0.01 ; \quad$ Group $\times$ Stimuli $\times$ Electrode: $\mathrm{F}(2,20)=0.68, p>0.9]$ and after presentation of the probe recognition items at P3 [Group $\times$ Electrode: $\mathrm{F}(2,20)=3.17, p<0.5$; Group $\times$ Stimuli $\times$ Electrode: $\mathrm{F}(2,20)=1.32, p>$ $0.2]$. At P5 however, amplitude levels were similar for all electrodes for both groups [Group $\times$ Electrode: $\mathrm{F}(2,20)=2.07, p>0.1]$, but there were differences between the stimulus conditions [Stimulus $\times$ Group $\times$ Electrode: $\quad \mathrm{F}(2,20)=3.16, \quad p<0.05]$. Separate ANOVAs (Group $\times$ Electrode) for the two conditions showed similar amplitudes between the electrodes for the words $[\mathrm{F}(2,20)=0.66, p>0.9]$ but different levels at $\mathrm{FZ}, \mathrm{CZ}$, and $\mathrm{PZ}$ for the spatial abstract designs $[\mathrm{F}(2,20)=4.82, p<0.01]$.

\section{ERP latency delay in PD}

Amabile et al. (1990) found delayed P3 latencies in PD. These results were confirmed in the present study with slower ERP latencies for the PD group than for the control group (Probe recognition items: Averages of FZ, CZ and PZ electrodes were $85 \mathrm{~ms}$ slower at P3 and $40 \mathrm{~ms}$ slower at P5 for the PD group; Memory set items: Averages of FZ, CZ and PZ electrodes were $125 \mathrm{~ms}$ slower for the PD group). MANOVAs on the PZ electrode (Group $\times$ Stimuli $\times$ Position) confirm the differences showing large main group effects at encoding $[F(1,10)=$ $40.88, p<0.01]$, both for the P3 $[\mathrm{F}(1,10)=20.23$, $p<0.01]$ and P5 components $[\mathrm{F}(1,10)=24.85$, $p<0.01]$ after presentation of the probe. These results were similar for all list positions at encoding $[\mathrm{F}(2,20)=0.22, p>0.8]$ and for the $\mathrm{P} 3$ component after presentation of the probe $[\mathrm{F}(3,30)=0.31$, $p>0.8]$, and at P5 $[\mathrm{F}(3,30)=0.43, p>0.7]$. None of the experimental parameters interact, and the latencies were not analysed further.

\section{DISCUSSION}

The present paradigm is a variation of the 'recency recognition' paradigm with the added requirement that the exact sequential position of an item must be indicated. The finding from our previous studies has been that a list position curve can be found in this paradigm with a primacy and recency effect analogous to that observed in ordinary recall paradigms. The results do not identify a behavioural deficit in $\mathrm{PD}$ related to list position recognition for abstract spatial patterns, but indicate individual variation in the recency performance of the PD group on a task which is more demanding on working memory. Cooper et al. (1993) did not find evidence of a generalized temporal ordering deficit for verbal information in $\mathrm{PD}$ patients, which corresponds to the present study. They concluded that all evidence of neuropsychological deficits in newly diagnosed and unmedicated PD patients was found with tests that put strains on working memory. There is no conclusive evidence of serial position related abnormalities in the performance of either words or abstract spatial designs for the PD patients. Accuracy was lower for spatial abstract patterns than for words in both PD and controls, as for young and older subjects in the study of Korsnes and Magnussen (1996). Both primacy and recency effects were present for the control group and a primacy effect for the PD group, 


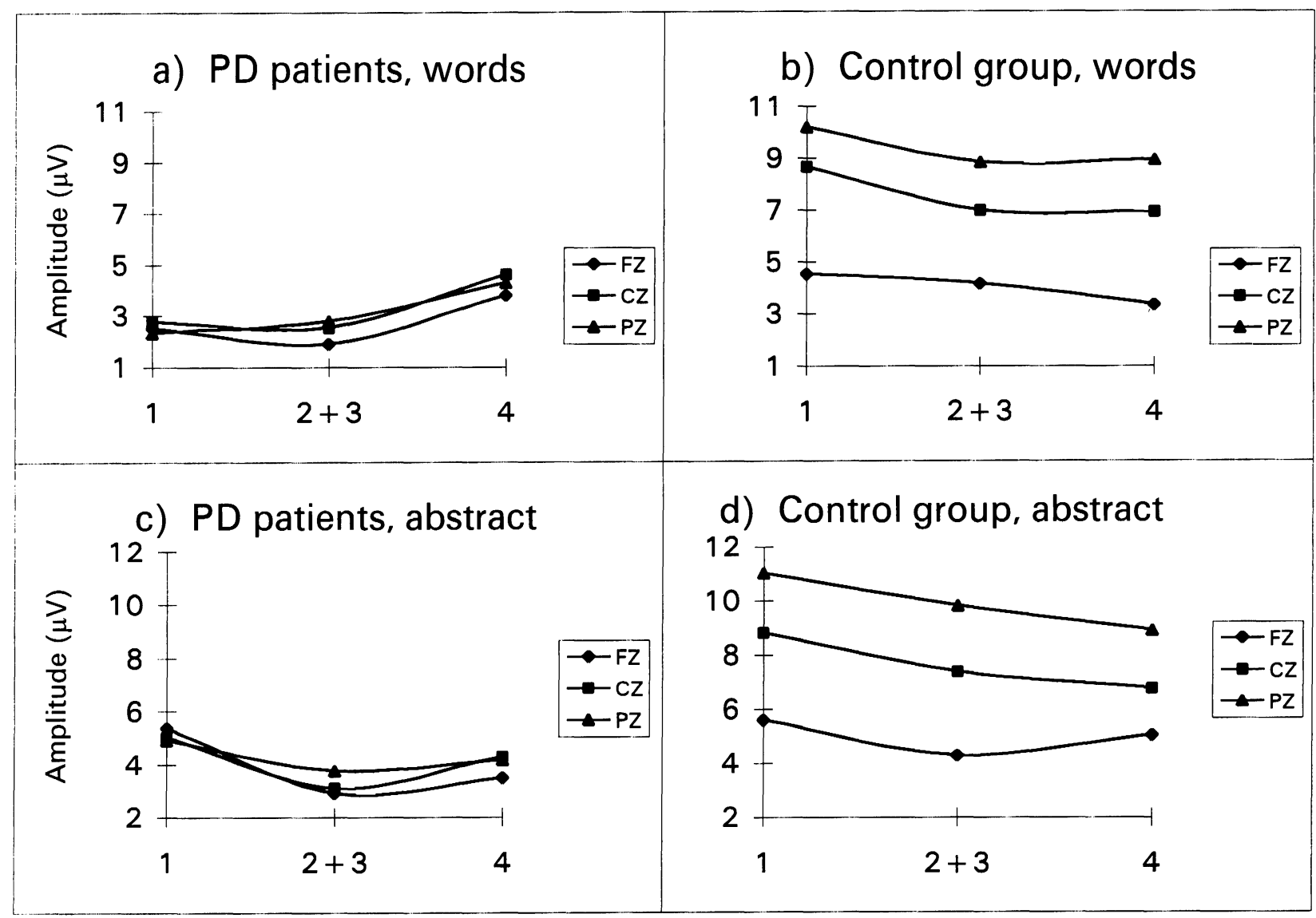

List Position

List Position

FIG. 7. ERPs to probes after presentation of the memory set items for the two groups under the two conditions. The highest positive amplitude within a window from $200-450 \mathrm{~ms}$ was recorded.

but deviant performance on the last list position did not give conclusive evidence for a deficit in recency performance for the spatial abstract designs. The most surprising result was that the PD group showed a high no-response rate, indicating that they had general problems performing the task of pressing one out of four buttons after recognition presentation of a pictorial probe. Overall they failed to respond in $29 \%$ of all trials. Previous studies (i.e. Ransmayr et al., 1990) did not report general problems for PD patients in responding to probe items in memory scan tasks. The present study differs in requesting explicit temporal order recognition, and may therefore put higher demands on working memory.

The ERP results are consistent with previous studies in showing general ERP abnormalities in PD. The generally delayed P3 and P5 ERP latencies for the PD group and lower amplitude for P3, confirm the results of Amabile et al. (1990), who suggested that this is an effect of a dopaminergic system deficit, because they observed a generally faster P3 in healthy subjects and PD patients after intake of $\mathrm{L}$-dopa medication. The small sample did not allow for a statistical comparison between medicated and unmedicated patients. Higher amplitudes were found for the control group than for the PD patients for words and abstract spatial designs at P3 both at encoding and after presentation of the probe, particularly at the PZ electrode. The amplitude level was similar for all electrodes for $\mathrm{PD}$, but the amplitudes at FZ were lower than at $\mathrm{CZ}$ and highest at PZ for the control group. At P5, the amplitude level was similar for all electrodes for both groups, in contrast to the results at P3. The difference in topography lends some support to our decision to analyse a P5 as distinct from a $\mathrm{P} 3$ component.

The behavioural results are in contrast to the ERP list position results, where differences in position 
effects between the groups were found for the P5 ERPs recorded after presentation of the probe. Compared to previous research (Paller et al., 1987, 1988; Rugg et al., 1989), the present results indicate that ERP component most likely to be sensitive to memory effects is also sensitive to serial position effects. Moreover, the group differences that could not be shown in the behavioural data are significant in the ERPs, indicated by an interaction between group and serial position. List position differences were also found between the two conditions for the PD patients, but not for the control group. The group $\times$ position interaction at P5 may indicate that ERP is a more sensitive measure than accuracy. Thus, taking the ERP data into consideration, evidence is found both for a general processing difference between the groups and a serial-position related change in $\mathrm{PD}$. This conclusion accords with previous behavioural data (Cooper et al., 1993; Della Sala et al., 1987). We find possible ERP differences in the serial position function for both words and abstract spatial designs at P3. Based upon the accuracy results and the ERP indicators, we can therefore not conclude that the serial list functions in our PD group is unaffected for the verbal material (Cooper et al., 1993), and a possible recency deficit is indicated for the spatial abstract designs. These results align with the impaired recency discrimination for nouns, found by Sagar et al. (1988) when they tested their subjects after short intervals, and the impairment is perhaps more prominent for abstract stimuli.

Given the evidence of visuo-spatial and attentional processing deficits in PD reviewed in the introduction it is of central importance to ascertain if the memory-related findings can be accounted for by deficits in encoding of the stimulus material. The ERP results for memory set items confirm the hypothesis of a processing deficit in the PD group, and the memory set data indicate both a general processing difference and a group interaction involving serial position. The small number of subjects does not allow an analysis predicting performance on the basis of memory set ERP, but the evidence is that processing differences at the time of encoding may account for short-term memory effects. Indirectly the data are consistent with an interpretation of cognitive deficit stressing limited attention resources in PD (Boller et al., 1984; Bradley et al., 1989; Cooper et al., 1992; Filoteo et al., 1994).

\section{Acknowledgements}

This research was supported by grant no $377.92 / 016$ by the Norwegian Research Council.

\section{REFERENCES}

Amabile G, Fattapposta F and Pierelli F (1990) Evoked potentials in Parkinson's disease: sensory and cognitive aspects. A review. Journal of Psychophysiology, 4, 122-155.

Begleiter H, Porjesz B and Wang W (1993) A neurophysiologic correlate of visual short-term memory in humans. Electroencephalography and Clinical Neurophysiology, 87, 46-53.

Boller F, Passafiume D, Keefe NC, Rogers K, Morrow L and Kim Y (1984) Visuospatial impairment in Parkinson's disease: role of perceptual and motor factors. Archives of Neurology, 41, 485-490.

Bradley VA, Welch JL and Dick DJ (1989) Visuospatial working memory in Parkinson's disease. Journal of Neurology, Neurosurgery and Psychiatry, 52, 1228-1235.

Breen KE (1993) Recall and recognition memory in Parkinson's disease. Cortex, 29, 91-102.

Cooper JA, Sagar HJ, Jordan N, Harvey NS and Sullivan EV (1991) Cognitive impairment in early untreated Parkinson's disease and its relationship to motor disability. Brain, 114, 2095-2122.

Cooper JA, Sagar HJ, Doherty SM, Jordan N, Tidswell PT and Sullivan EV (1992) Different effects of dopaminergic and anticholinergic therapies on cognitive and motor function in Parkinson's disease: a follow-up study of untreated patients. Brain, 115, 1701-1725.

Cooper JA, Sagar HJ and Sullivan EV (1993) Short-term memory and temporal ordering in early Parkinson's disease: effects of disease chronicity and medication. Neuropsychologia, 31, 933-949.

Craik FIM (1968) Two components in free recall. Journal of Verbal Learning and Verbal Behavior, 7, 996-1004.

Della Sala S, Di Lorenzo G, Giordano A and Spinnler H (1986) Is there a specific visuo-spatial impairment in parkinsonians? Journal of Neurology, Neurosurgery and Psychiatry, 49, 1258-1265.

Della Sala S, Pasetti C and Sempio P (1987) Deficit of the "primacy effect" in Parkinsonians interpreted by means of the working memory model. Swiss Archives of Neurology, Neurosurgery and Psychiatry, 138, 5-14.

Filoteo VJ, Delis DC, Demadura TL, Salmon DP, Roman MJ and Shults CW (1994) Abnormally rapid disengagement of covert attention to global and local stimulus levels may underlie the visuoperceptual impairment in Parkinson's patients. Neuropsychology, 8, 210-217.

Flowers KA, Pearce I and Pearce JMS (1984) Recognition memory in Parkinson's disease. Journal of Neurology, Neurosurgery and Psychiatry, 47, 1174-1181.

Glanzer M and Cunitz AR (1966) Two storage mechanisms in free recall. Journal of Verbal Learning and Verbal Behaviour, 17, 455463.

Gomer FE, Spicuzza RJ and O'Donell RD (1976) Evoked potential correlates of visual item recognition during memory-scanning tasks. Physiological Psychology, 4, $61-65$.

Karayanidis F, Andrews S, Ward PB and McConaghy N (1993) Event-related potentials and repetition priming in young, middle-aged and elderly normal subjects. Cognitive Brain Research, 1, 123-134.

Kausler DH (1991) Experimental Psychology, Cognition, and Human Aging. 2nd. Edition. Springer-Verlag.

Korsnes MS and Gilinsky SA (1993) Aging and serial list picture memory. Perceptual and Motor Skills, 76, 10111014. 
Korsnes MS and Magnussen S (1996) Age comparisons of list position effects in visual short-term memory. Acta Psychologica, 94 (2), (in press).

Korsnes MS, Magnussen S and Reinvang I (1996) Serial position effects in visual short-term memory for words and spatial patterns. Scandinavian Journal of Psychology, 37, 62-73.

Levin BE, Libre MM and Weiner W (1989) Cognitive impairments associated with early Parkinson's disease. Neurology, 39, 557-561.

Lewandosky S and Murdock BB (1989) Memory for serial order. Psychological Review, 96, 25-27.

Paller KA, Kutas M and Mayes AR (1987) Neural correlates of encoding in an incidental learning paradigm. Electroencephalography and Clinical Neurophysiology, 67, 360-371.

Paller KA, McCarthy G and Wood CC (1988) ERPs predictive of subsequent recall and recognition performance. Special issue: Event related potential investigations of cognition. Biological Psychology, 26, 269-276.

Patterson JV, Pratt H and Starr A (1991) Event-related potential correlates of the list position effect in shortterm memory. Electroencephalography and Clinical Neurophysiology, 78, 424-437.

Pratt H, Michalewski HJ, Barret G and Starr A (1989) Brain potentials in a memory-scanning task. I. Modality and task effects on potentials to the probes. Electroencephalography and Clinical Neurophysiology, 72, 407421.

Ransmayr G, Bitschnau W, Schmidhuber-Eiler B, Berger W, Karamat E, Poewe W and Kemmler GW (1990) Slowing of high-speed memory scanning in Parkinson's disease is related to the severity of parkinsonian motor symptoms. Journal of Neural Transmission, 2, 265-275.

Reitan RM and Boll TJ (1971) Intellectual and cognitive function in Parkinson's disease. Journal of Consulting and Clinical Psychology, 37, 364-369.

Rugg MD, Pearl S, Walker P, Roberts RC and Holdsstock JS (1994) Word repetition effects on event-related potentials in healthy young and old subjects, and in patients with Alzheimer type dementia. Neuropsychologia, 32, 381-398.

Sagar HJ, Cohen NJ, Gabrielli JDE, Corkin S and Growdon J (1985) Specific cognitive deficits in Parkinson's disease. Journal of Clinical and Experimental Neuropsychology, 7, 158.

Sagar HJ, Sullivan EV, Gabrielli JDE, Corkin S and Growdon JH (1988) Temporal ordering and short-term memory deficits in Parkinson's disease. Brain, 111, 525539.

Sahakian BJ, Morris RG, Evenden JL, Herald A, Levy R, Philpot M and Robbins TW (1988) A comparative study of visuospatial memory and learning in Alzheimer-type dementia and Parkinson's disease. Brain, 11, 695-718.

Semlitsch HV, Ander R, Schuster P and Presslich O (1986) A solution for reliable and valid reduction of ocular artifacts, applied to the P300 ERP. Psychophysiology, 23, 695-703.

Sullivan EV and Sagar HJ (1989) Nonverbal recognition and recency discrimination deficits in Parkinson's and Alzheimer's disease. Brain, 112, 1503-1517.

Talland GA (1969) Cognitive functions in Parkinson's disease. Journal of Nervous and Mental Disease, 135, 196205.

Taylor AE, Saint-Cyr JA and Lang AE (1986) Frontal lobe dysfunction in Parkinson's disease: the cortical focus of neostriatal outflow. Brain, 109, 845-883.

Wright AA, Santiago HC, Sands SF, Kendrick DF and Cook RG (1985) Memory processing of serial lists by pigeons, monkeys and people. Science, 229, 287-289. 


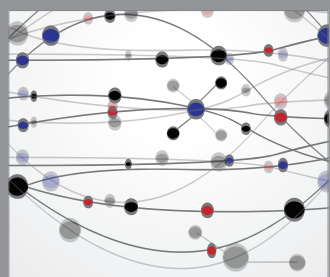

The Scientific World Journal
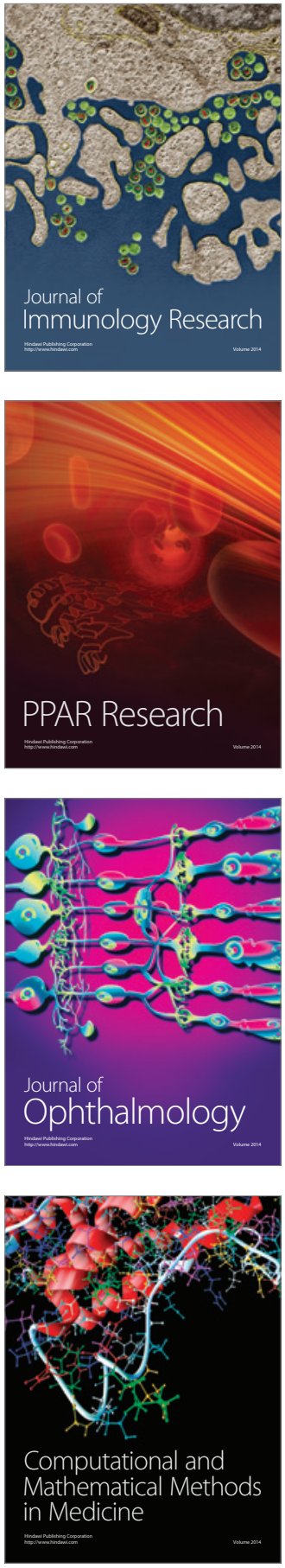

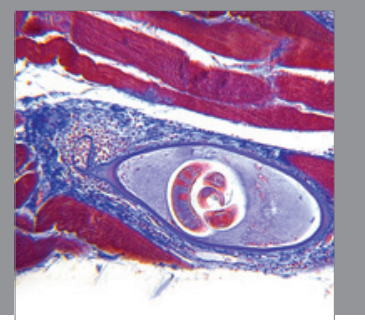

Gastroenterology

Research and Practice
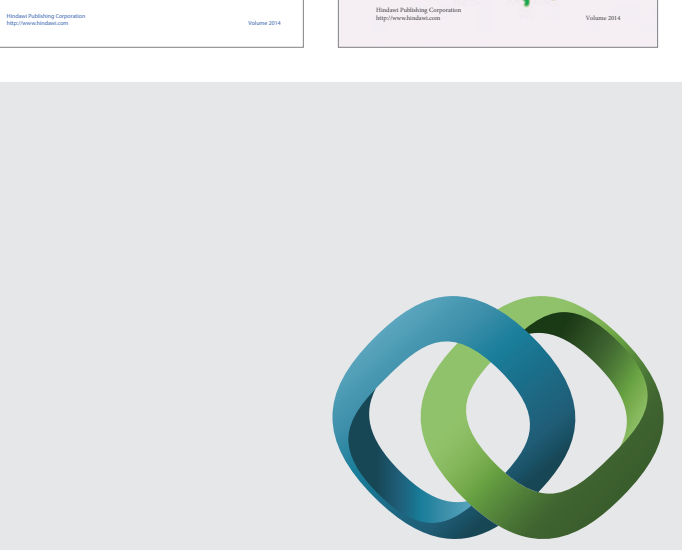

\section{Hindawi}

Submit your manuscripts at

http://www.hindawi.com
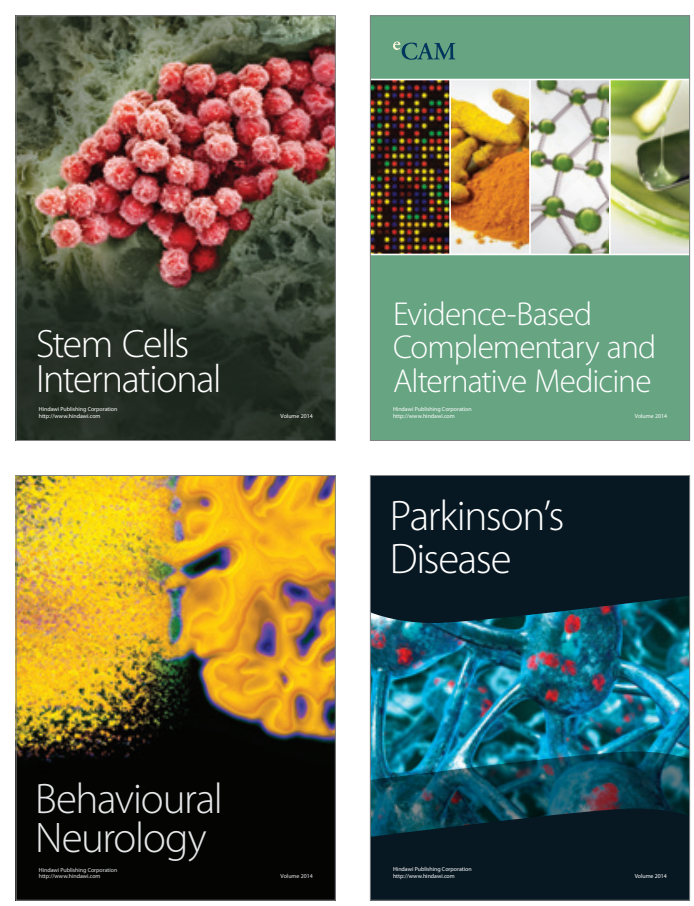

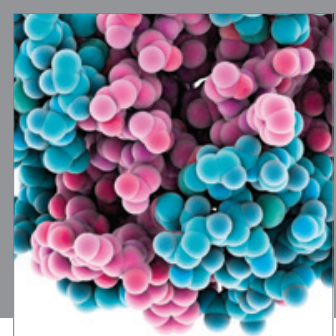

Journal of
Diabetes Research

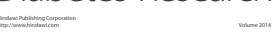

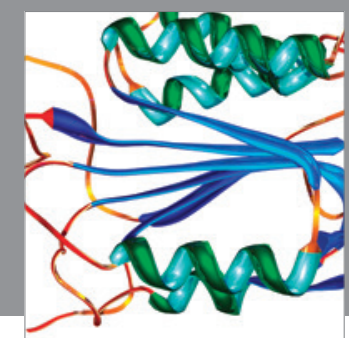

Disease Markers
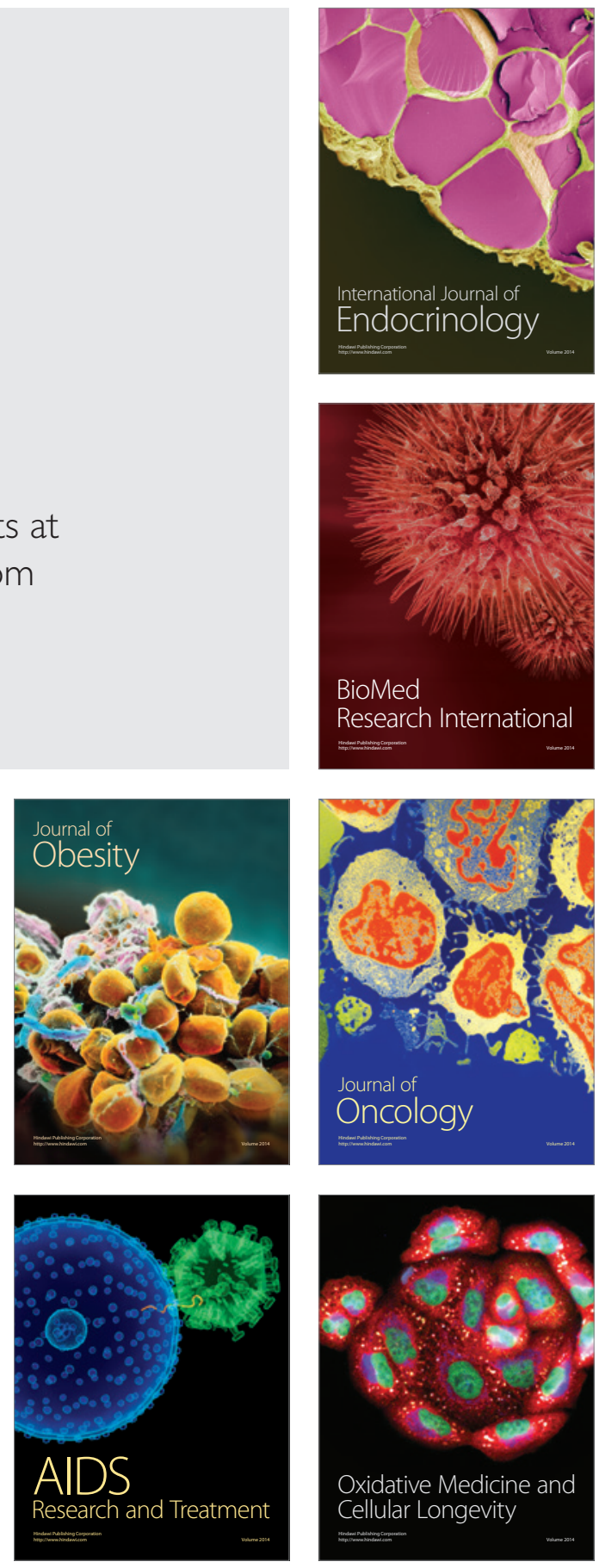